\title{
Superior Suprarenal Artery
}

National Cancer Institute

\section{Source}

National Cancer Institute. Superior Suprarenal Artery. NCI Thesaurus. Code C52736.

Either of two arteries (left and right) arising from the inferior phrenic artery that supply the corresponding adrenal gland. 